\title{
ZNF259 inhibits non-small cell lung cancer cells proliferation and invasion by FAK-AKT signaling
}

This article was published in the following Dove Press journal:

Cancer Management and Research

\section{Yuemei Shan ${ }^{1,2}$ \\ Wei Cao' \\ Tao Wang' \\ Guiyang Jiang ${ }^{3}$ \\ Yong Zhang ${ }^{4}$ \\ Xianghong Yang'}

'Department of Pathology, Shengjing Hospital of China Medical University, Shenyang, China; ${ }^{2}$ Department of Applied Technology, Institute of Technology of China Medical University, Shenyang, China; ${ }^{3}$ Department of Pathology, First Affiliated Hospital and College of Basic Medical Sciences, China Medical University, Shenyang, China; ${ }^{4}$ Department of Pathology, Cancer Hospital of China Medical University, Shenyang, China
Correspondence: Xianghong Yang Department of Pathology, Shengjing Hospital of China Medical University, Number 36 Sanhao Street, Shenyang II 0004, China

Tel +86 I 894025 I640

Fax +8602423892617

Email xhyang4933@vip.sina.com
Background: Zinc finger protein 259 (ZNF259) is known to play essential roles in embryonic development and cell cycle regulation. However, its expression pattern and clinicopathological relevance remain unclear.

Materials and methods: A total of 114 lung cancer specimens were collected. The ZNF259 expression was measured between the lung cancer tissues and the adjacent normal lung tissues by immunohistochemical staining and Western blotting. Moreover, the correlation of ZNF259 expression with clinicopathological features was analyzed in 114 cases of lung cancer. Additionally, ZNF259 was depleted in the lung cancer cells in order to analyze its effect in the lung cancer. Results: Immunohistochemical staining of 114 lung cancer specimens revealed significantly lower ZNF259 expression in lung cancer tissues than in adjacent normal lung tissues $(53.5 \% \mathrm{vs}$ $71.4 \%, P<0.001)$. In addition, ZNF259 downregulation was significantly associated with larger tumor size $(P=0.001)$, advanced TNM stage $(P=0.002)$, and positive lymph node metastasis $(P=0.02)$. Western blotting of 20 paired lung cancer samples revealed lower ZNF259 protein levels in lung cancer tissues than in those of corresponding normal lung tissues $(P=0.0032)$. Depletion of ZNF259 resulted in enhanced levels of p-FAK and p-AKT, CyclinD1, and MMP2, which in turn increased the proliferation and invasion of lung cancer cells. The effects of ZNF259 depletion were reversed by treatment with specific FAK or AKT inhibitors.

Conclusion: ZNF259 depletion is correlated with the development of non-small cell lung cancer (NSCLC) and serves as a predictor of adverse clinical outcome in NSCLC patients. The inhibitory effect of ZNF259 on proliferation and invasion can be attributed to downregulation of CyclinD1 and MMP2 via inactivation of the FAK-AKT pathway.

Keywords: ZNF259, NSCLC, FAK signaling, AKT signaling, proliferation, invasion

\section{Introduction}

Lung cancer is currently one of the most common malignant tumors worldwide. ${ }^{1,2}$ Detailed understanding of the mechanisms involved in the invasion and proliferation of lung cancer is important for the identification of new targets for clinical therapy and for improving clinical treatment strategies.

Zinc finger protein 259 (ZNF259) is also known as zinc finger protein 1 (ZPR1). Gangwani et al previously demonstrated that ZNF259 plays essential roles in early embryonic development, and that ZNF259 deficiency results in growth cone retraction, axonal defects, and apoptosis. ${ }^{3-5}$ A study by Gangwani also confirmed that ZNF259 localizes both in the cytoplasm and nucleus of HeLa cells and induces major changes in subcellular distribution during proliferation. ${ }^{6} \mathrm{ZNF} 259$ has been shown to translocate 
to the nucleus during the $\mathrm{S}$ phase and accelerate cell cycle progression. $^{6}$

However, to date, little is known on about the expression patterns and molecular mechanisms underlying the effects of ZNF259, especially in human lung cancer. In this pilot study, we performed immunohistochemistry to investigate the effects of ZNF259 in human non-small cell lung cancer (NSCLC) samples. ZNF259 showed strong expression in the cytoplasm in noncancerous tissues, but weak expression in the cytosol in NSCLC specimens. These observed expression patterns were different from the results reported by Gangwani, indicating that ZNF259 may play distinct biologic roles in malignant tumors.

In this study, we explored the protein levels and subcellular distributions of ZNF259 in both lung cancer tissues and cell lines, as well as their corresponding clinicopathologic relevance. We also investigated the effects of ZNF259 on the proliferation and invasiveness of NSCLC cell lines upon ZNF259 overexpression or depletion. Results showed that ZNF259 suppressed the proliferation and invasion of NSCLC cells by inhibiting activation of the FAK-AKT signaling pathway.

\section{Materials and methods}

\section{Patients and specimens}

This study was approved by the local institutional review board of the China Medical University. The internal review board's approval and patients' informed consent for this study were also obtained. Tissue samples were obtained from 114 patients ( 86 males and 28 females) who underwent complete surgical excision at the Shengjing Hospital of China Medical University between 2012 and 2014. All patients were diagnosed with NSCLC, and 35 of 114 cases had corresponding noncancerous lung tissues. No neoadjuvant radiotherapy or chemotherapy was performed before surgery. Histologic diagnosis and grading were performed according to the 2015 World Health Organization classification of lung tumors. ${ }^{7}$ All 114 specimens were analyzed based on histologic subtype, differentiation, and tumor stage. Tumor staging was performed according to the seventh edition of the International Union against Cancer TNM Staging System for Lung Cancer. $^{8}$ The median age of the 114 patients was 57 years (range: 29-79 years). Out of the 114 patients, 68 patients were at least 57 years old, while 46 patients were younger than 57 years. Samples included 42 squamous cell lung carcinomas, 54 lung adenocarcinomas, and 18 other histologic type lung cancers. A total of 16 tumors were classified as well differentiated, while 44 and 34 tumors were considered moderately and poorly differentiated, respectively. Lymph node metastases were present in 52 of the 114 cases. Our cohort included 61 cases in stages I-II and 53 cases in stage III.

In addition, fresh tissue specimens were available from 20 cases, which included both tumor and paired noncancerous tissues. Samples were stored at $-80^{\circ} \mathrm{C}$ immediately after resection for subsequent protein extraction.

\section{Immunohistochemistry}

Samples were fixed in 10\% neutral formalin, embedded in paraffin, and sliced into $4 \mu \mathrm{m}$ thick sections. Immunostaining was performed following the streptavidin-peroxidase method. Sections were incubated with a monoclonal rabbit anti-ZNF259 antibody (1:100, ab134970; Abcam, Cambridge, UK) at $4{ }^{\circ} \mathrm{C}$ overnight, followed by incubation with biotinylated goat anti-rabbit IgG secondary antibody. After washing, the sections were incubated with horseradish peroxidase-conjugated streptavidin-biotin (Ultrasensitive; MaiXin, Fuzhou, China) and developed using 3,3-diaminobenzidine tetrahydrochloride (MaiXin). Finally, the samples were lightly counterstained with hematoxylin, dehydrated in alcohol, and mounted. Two investigators blinded to the clinical data performed semi-quantitative scoring of the slides by evaluating the staining intensity and determining the percentage of stained cells in representative areas. Staining intensity was scored as 0 (no signal), 1 (weak), 2 (moderate), or 3 (high). The percentage of positively stained cells was scored as 0 (no signal), 1 (1\%-25\%), 2 $(26 \%-50 \%), 3(51 \%-75 \%)$, or $4(76 \%-100 \%)$. Final scores, which ranged from 0 to 12 , were obtained by multiplying the intensity and percentage scores. Tumors with scores $\geq 4$ were considered to exhibit positive ZNF259 expression. Tumor samples with scores between 1 and 3 were considered to exhibit weak ZNF259 expression, whereas those with scores of 0 were considered to have negative expression. In the statistical analysis, the expression of ZNF259 was divided into two groups (overexpression and low expression). The overexpression group included tumors with scores $\geq 4$, and the low expression group included tumors with scores between 0 and 3 .

\section{Cell culture}

The HBE cell line was obtained from the American Type Culture Collection (ATCC; Manassas, VA, USA). The A549, H460, SPC, and H1299 cell lines were obtained from the Shanghai Cell Bank (Shanghai, China). The LK2 cell line was a gift from Dr Hiroshi Kijima (Department of Pathology and Bioscience, Hirosaki University Graduate School 
of Medicine, Japan). The human lung ADC Anip973 and AGZY83a cell lines were purchased from Shanghai Bioleaf Biotech Co., Ltd (Shanghai, China) and stored in the Department of Pathology, Harbin Medical University. All cell lines were verified by short tandem repeat DNA profiling. Cells were frozen upon receipt, and individual aliquots were typically passaged 10 times for analysis. All cells were cultured in RPMI 1640 (Thermo Fisher Scientific, Waltham, MA, USA) supplemented with $10 \%$ fetal bovine serum (FBS; Thermo Fisher Scientific), $100 \mathrm{IU} / \mathrm{mL}$ penicillin (Sigma, St Louis, MO, USA), and $100 \mu \mathrm{g} / \mathrm{mL}$ streptomycin (Sigma). Cells were passaged every other day using $0.25 \%$ trypsin (Thermo Fisher Scientific).

\section{Western blotting}

Total protein was extracted using a lysis buffer (Pierce, Rockford, IL, USA) and quantified with the Bradford method. ${ }^{9}$ Total proteins $(50 \mu \mathrm{g})$ were separated by $10 \%$ sodium dodecyl sulfate-polyacrylamide gel electrophoresis and subsequently transferred onto polyvinylidene fluoride membranes (EMD Millipore, Billerica, MA, USA). Membranes were incubated overnight at $4{ }^{\circ} \mathrm{C}$ with the following primary antibodies: ZNF259 (1:500, Abcam), GAPDH (1:5000, Sigma), Myctag, FLAG, Snail, MMP2, MMP9, cyclin D1, cyclin E1, CDK4, CDK6, p-AKT-Thr308, AKT, p-p38, p38, p-JNK, JNK p-FAK-Tyr397, FAK (1:1000; Cell Signaling Technology, Danvers, MA, USA), and E-cadherin (1:1000; BD Transduction Laboratories, Lexington, KY, USA). FAK inhibitor (PF-562271) was obtained from Selleck Chemicals (Houston, TX, USA). AKT inhibitor LY294002 was obtained from Cell Signaling Technology. Membranes were washed and subsequently incubated with peroxidase-conjugated anti-mouse or anti-rabbit IgG (Santa Cruz Biotechnology) at $37^{\circ} \mathrm{C}$ for 2 $\mathrm{h}$. Bound proteins were visualized using electrochemiluminescence (Pierce) and detected with a bio-imaging system (DNR Bio-Imaging Systems, Jerusalem, Israel).

\section{Plasmid transfection and siRNA treatment}

Plasmids pCMV6-ddk-myc and pCMV6-ddk-myc-ZNF259 were purchased from Origene (RC205721; Origene, Rockville, MD, USA). ZNF259-siRNA (sc-35282) and negative control-siRNA (sc-37007) were purchased from Santa Cruz Biotechnology. Transfection was carried out using the Lipofectamine 3000 reagent (Thermo Fisher Scientific) according to the manufacturer's instructions. Cells were harvested within $48 \mathrm{~h}$ after plasmid transfection. For siRNA treatment, cells were harvested after $72 \mathrm{~h}$.

\section{Matrigel invasion assay}

Cell invasion assay was performed using a 24-well transwell chamber with $8 \mu \mathrm{m}$ pores (Costar, Cambridge, MA, USA). Inserts were coated with $20 \mu \mathrm{L}$ of Matrigel (1:3 dilution; BD Biosciences, San Jose, CA, USA). At 48 h after transfection, cells were trypsinized, and $3 \times 10^{5}$ cells in $100 \mu \mathrm{L}$ of serumfree medium were transferred to the upper Matrigel chamber for $18 \mathrm{~h}$. Media supplemented with 10\% FBS were added to the lower chamber as a chemoattractant. After incubation, cells that passed through the filter were fixed with $4 \%$ paraformaldehyde and stained with hematoxylin. Invasive cells were microscopically counted in 10 randomly selected high-power fields.

\section{Wound healing assay}

In cultures with cell densities below $90 \%$, wounds were created in confluent areas using a $200 \mu \mathrm{L}$ pipette tip at $48 \mathrm{~h}$ after transfection. Wound healing within the scrape line was observed at varying time points, and representative scrape lines for each cell line were photographed. Duplicate wells were examined for each condition, and each experiment was repeated three times. Optical wound distances were measured using ImageJ software (National Institute of Health, Bethesda, MD, USA).

\section{MTT assay}

At $24 \mathrm{~h}$ after transfection, cells were plated in 96-well plates in media containing $10 \%$ FBS at about 3000 cells per well. For quantitation of cell viability, cultures were stained after 4 days using the MTT assay. Briefly, $20 \mu \mathrm{L}$ of $5 \mathrm{mg} / \mathrm{mL}$ MTT solution was added to each well and incubated for $4 \mathrm{~h}$ at $37^{\circ} \mathrm{C}$ Next, the culture media were removed from each well, and the resulting MTT formazan product was solubilized in $150 \mu \mathrm{L}$ of DMSO. Results were quantitated spectrophotometrically at the wavelength of $570 \mathrm{~nm}$.

\section{Colony formation assay}

A549 cells were transfected with pCMV6 or pCMV6ZNF259 plasmids, negative control, or ZNF259-siRNA for $48 \mathrm{~h}$. Afterward, the cells were placed in three $6 \mathrm{~cm}$ cell culture dishes (1000 per dish for A549 cells) and incubated for 12 days. Plates were washed with PBS and stained with Giemsa. The number of colonies comprising more than 50 cells was manually counted under a microscope.

\section{Statistical analysis}

SPSS version 22.0 for windows (IBM Corporation, Armonk, NY, USA) was used for all statistical analyses. Pearson's chi- 
square test was used to evaluate correlations between ZNF259 and clinicopathologic features. Image analyses of Western blotting results and invasion assay results were performed using Mann-Whitney $U$ test. Statistical significance was considered at $P<0.05$.

\section{Results}

\section{ZNF259 expression in NSCLC specimens and cell lines}

Immunohistochemistry was initially performed to evaluate the expression and subcellular localization of ZNF259 in 114 NSCLC samples. Positive expression was mainly observed in bronchial ciliated columnar epithelial cells. Weak and negative expression was mainly observed in alveolar epithelial cells and glands around the trachea. Results revealed that ZNF259 was strongly expressed in the cytoplasm of peritumoral tissues (Figure 1Aa, b). However, ZNF259 showed weak or negative cytosolic expression in NSCLC specimens (Figure 1Ac-e). The positive rate of ZNF259 in noncancerous tissues $(71.4 \%$, $25 / 35)$ was evidently higher than that in cancerous samples (53.5\%, 61/114, $P<0.001$; Figure 1Af). Subsequent statistical analysis indicated that reduced ZNF259 expression was significantly correlated with tumor size $(P=0.001)$, TNM stage $(P=0.002)$, and lymph node metastasis $(P=0.02)$. However, ZNF259 expression was not correlated with age, gender, differentiation, and histologic type $(P>0.05$; Table 1$)$.

Then, we also measured ZNF259 protein levels in fresh NSCLC specimens. There were 2 cases that had higher, 12 cases that had lower, and 6 cases that had similar ZNF259 protein expression in NSCLC tissues compared with the adjacent noncancerous lung according to Western blotting results (Figure 1B). Based on Western blotting results, normalized protein levels of ZNF259 in normal lung tissues (Mean \pm SD: $0.9183 \pm 0.4242$ ) were evidently higher than those in NSCLC samples (Mean \pm SD: $0.5938 \pm 0.3276, P=0.0032$; Figure 1B, C). We also explored ZNF259 expression in NSCLC cell lines, and results revealed that ZNF259 expression was lower in seven out of eight NSCLC cells (except for H460; Figure 1D).

\section{ZNF259 suppressed NSCLC cell proliferation}

Next, we analyzed ZNF259 overexpression or siRNA knockdown in A549 cells (Figure 2A). MTT and colony formation assay results indicated lower proliferation (Figure 2B) and colony formation ability (Figure 2C) of A549 cells upon ZNF259 overexpression. Accordingly, cell proliferation (Figure 2B) and colony formation ability (Figure 2C) increased upon ZNF259 siRNA knockdown. We then detected the expression levels of cell cycle-related proteins by Western blotting. Results suggested that cyclin D1 was downregulated or upregulated after ZNF259 overexpression or knockdown in A549 cells. However, cyclin E1, CDK4, and CDK6 showed no detectable changes in expression (Figure 2D).

\section{ZNF259 abolished NSCLC migration and invasion}

We also explored the effect of ZNF259 overexpression or depletion on NSCLC invasion and metastasis in A549 cells. Results of wound healing and transwell assay showed that the migration (Figure 3A) and invasion (Figure 3B) of A549 cells was abolished upon ZNF259 overexpression, but enhanced upon ZNF259 depletion by siRNA treatment. Western blotting results revealed that MMP2 was downregulated or upregulated following ZNF259 overexpression or depletion, respectively. By contrast, the expression of other proteins, such as MMP9, Snail, and E-cadherin, showed no significant changes (Figure 3C).

\section{ZNF259 inhibited NSCLC proliferation and invasion by suppressing FAK-AKT signaling}

Finally, we screened for key signaling pathway factors involved in decreasing MMP2 and cyclin D1. Results indicated reduced phosphorylation of FAK in Tyr397 and AKT in Thr308 upon ZNF259 overexpression in A549 cells (Figure $4 \mathrm{~A})$. On the other hand, phosphorylation of FAK and AKT was enhanced following siRNA interference of ZNF259 (Figure 4A). Other signaling pathway proteins showed no visible changes in expression (Figure 4A). We next validated the upregulation of MMP2 and cyclin D1 by activation of FAK and AKT signaling. Specific inhibitors of FAK (PF562271) or AKT (LY294002) were added to the medium after ZNF259 depletion in A549 cells. MMP2 and cyclin D1 expression levels were no longer upregulated upon the addition of PF-562271 and LY294002 (Figure 4B, C). AKT phosphorylation in Thr308 was also abolished by treatment with PF-562271 and LY294002. However, inhibition of AKT signaling did not alter the phosphorylation levels of FAK in Tyr397, indicating that FAK is an upstream factor of AKT signaling and was induced upon ZNF259 expression.

\section{Discussion}

ZNF259 was reported to be responsible for embryonic development and cell cycle regulation. ${ }^{3-6}$ However, ZNF259 expression patterns in malignant tumors remain to be 
A
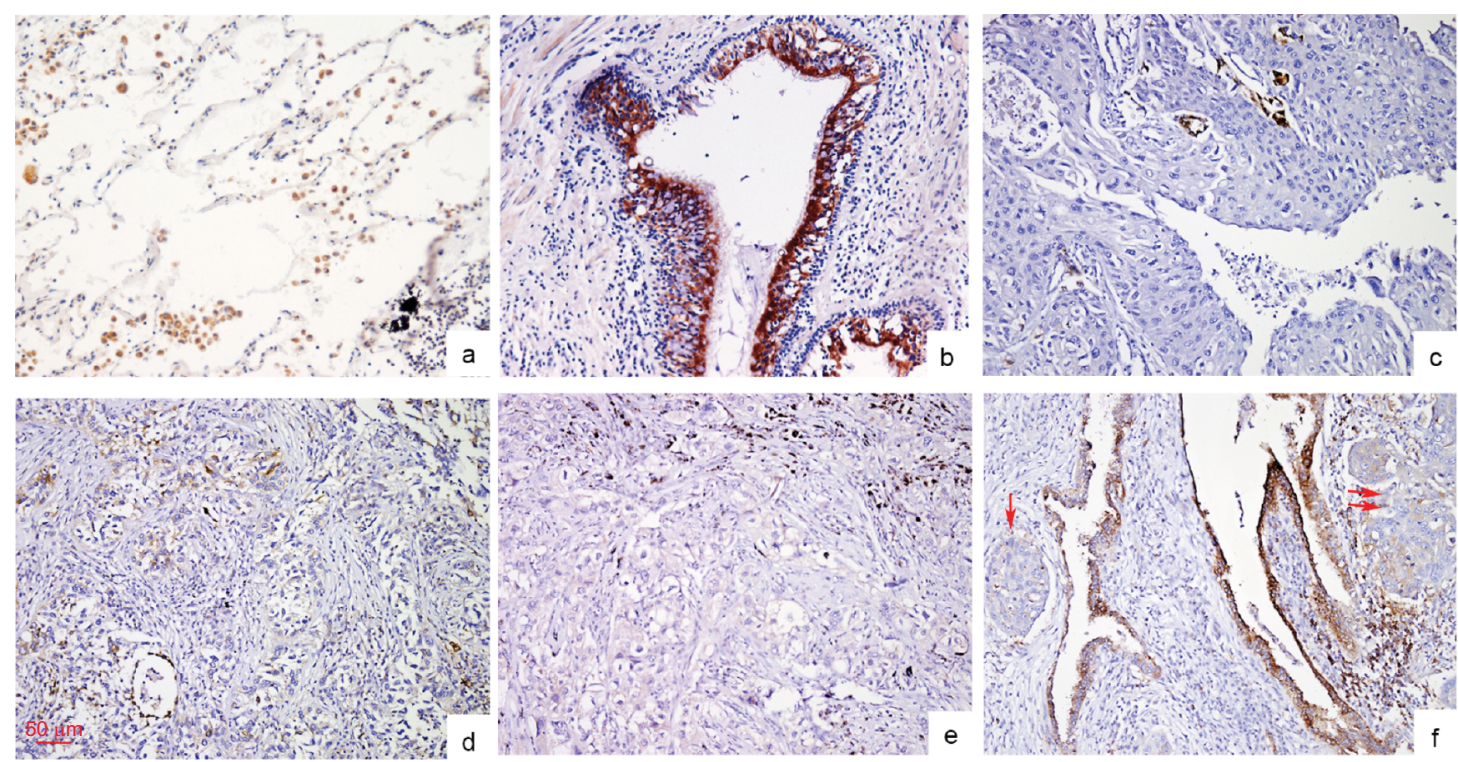

B
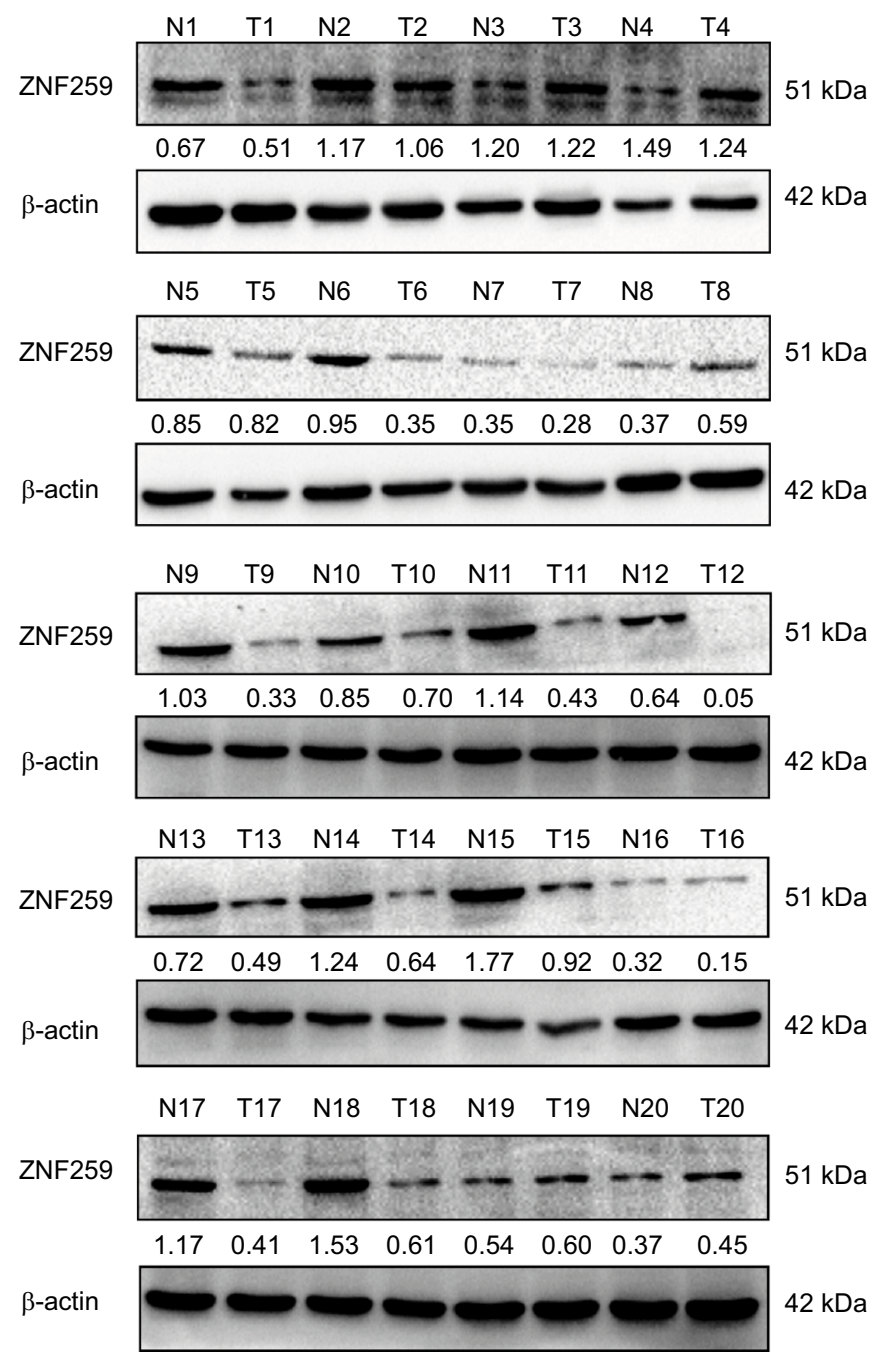

C

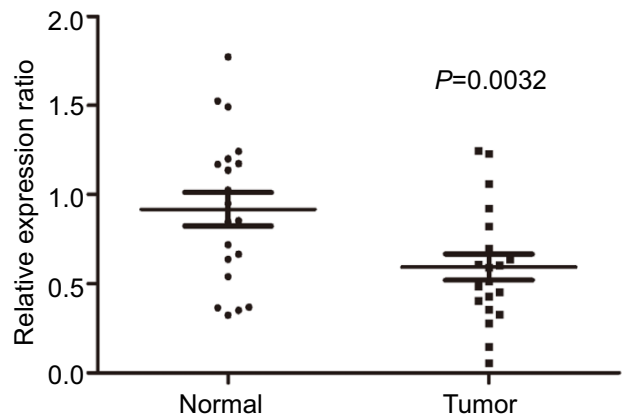

Figure I ZNF259 expression in NSCLC specimens and cell lines.

Notes: (A) Results revealed low ZNF259 expression in (a) alveolar epithelial and strong cytosolic expression in (b) normal bronchial epithelial cells. However, ZNF259 was weakly expressed in (c) lung squamous cell carcinoma, (d) adenocarcinoma, and (e) large cell lung carcinoma. (f) ZNF259 expression was significantly stronger in peritumoral tissues than in NSCLC specimens (indicated by red arrows). (B and C) Protein expression of ZNF259 in noncancerous tissues was considerably higher than that in NSCLC tissues. (D) ZNF259 expression in HBE cells was evidently higher than that in all of NSCLC cells examined, except for H460 cells. All studies were performed three times. Abbreviations: NSCLC, non-small cell lung cancer; ZNF259, zinc finger protein 259. 
Table I Correlation of ZNF259 expression with clinicopathologic features in II 4 cases of lung cancer

\begin{tabular}{|c|c|c|c|c|c|}
\hline \multirow[t]{2}{*}{ Features } & \multicolumn{5}{|l|}{ ZNF259 } \\
\hline & Number & Low expression & Overexpression & $\chi^{2}$ & $P$-value \\
\hline \multicolumn{6}{|l|}{ Gender } \\
\hline Male & 86 & 43 & 43 & 1.733 & 0.188 \\
\hline Female & 28 & 10 & 18 & & \\
\hline \multicolumn{6}{|l|}{ Age (years) } \\
\hline$<57$ & 46 & 18 & 28 & 1.68 & 0.251 \\
\hline$\geq 57$ & 68 & 35 & 33 & & \\
\hline \multicolumn{6}{|l|}{ Histology } \\
\hline Adenocarcinoma & 54 & 24 & 30 & 3.647 & 0.161 \\
\hline Squamous cell carcinoma & 42 & 17 & 25 & & \\
\hline Others & 18 & 12 & 6 & & \\
\hline \multicolumn{6}{|l|}{ Histology grade } \\
\hline GI & 16 & 6 & 10 & 0.307 & 0.858 \\
\hline G2 & 44 & 20 & 24 & & \\
\hline G3 & 34 & 15 & 19 & & \\
\hline \multicolumn{6}{|l|}{ Tumor size } \\
\hline $\mathrm{TI}+\mathrm{T} 2$ & 89 & 34 & 55 & 11.209 & $0.001 *$ \\
\hline $\mathrm{T} 3+\mathrm{T} 4$ & 25 & 19 & 6 & & \\
\hline \multicolumn{6}{|l|}{ Lymph node metastasis } \\
\hline No & 62 & 27 & 35 & 5.421 & $0.02 *$ \\
\hline Yes & 52 & 34 & 18 & & \\
\hline \multicolumn{6}{|l|}{ TNM stage } \\
\hline $\mathrm{I}+\mathrm{II}$ & 61 & 20 & 41 & 9.906 & $0.002 *$ \\
\hline III & 53 & 33 & 20 & & \\
\hline
\end{tabular}

Note: $* P<0.05$.

Abbreviation: ZNF259, Zinc finger protein 259.

investigated. Our study showed that ZNF259 was strongly expressed in the cytoplasm of noncancerous lung tissues, but weakly expressed in NSCLC samples. The expression level of ZNF259 in NSCLC cell lines (except for H460) was lower than that in normal bronchial epithelial cell line HBE, especially in H1299 cells harboring a p53-deletion mutant which is almost negative. We next asked whether the ZNF259 expression was influenced by $\mathrm{p} 53$, which should be further tested in future studies. Downregulation of ZNF259 in the cytoplasm was found to be significantly correlated with tumor size, TNM stage, and lymph node metastasis of NSCLC. Correspondingly, our results also suggested that ZNF259 inhibits the proliferation and the clone-forming, migration, and invasion abilities of NSCLC cells, and that ZNF259 downregulates cyclin D1 and MMP2 expression. Cyclin D1, one of the key proteins of G1/S checkpoint, could promote cell cycle progression from $\mathrm{G} 1$ to $\mathrm{S}$ phase. ${ }^{10-12}$ MMP2 could enhance the migration and invasion abilities of tumor cells. ${ }^{13,14}$ Thus, we hypothesized that ZNF259 can function as a tumor suppressor by inhibiting the expression of cyclin D1 and MMP2.

However, our results were different from previous reports. ZNF259 was initially identified as a cytoplasmic zinc finger protein that interacts with tyrosine kinase receptors, includ- ing epidermal growth factor receptor. ${ }^{15}$ Subsequent studies showed that the subcellular distribution of ZNF259 changes during cell proliferation; ZNF259 accumulates in the nucleus during the $\mathrm{S}$ phase to interact with eukaryotic translation elongation factor- 1 and promote cell cycle progression. ${ }^{6}$ However, in our study, nuclear expression of ZNF259 was not detected in noncancerous lung tissues and NSCLC tissues. We hypothesized that these contrasting results can be attributed to different histologic types, and that ZNF259 functions only in the cytoplasm of lung tissues. The varying effects of ZNF259 on the cell cycle may be caused by different subcellular localizations in different tissues, implying that cell cycle progression can be accelerated by nuclear ZNF259 but suppressed by cytosolic ZNF259. To verify our hypothesis, further experiments need to be performed in other tissues. The detailed mechanisms underlying the effects of ZNF259 also warrant further study.

To verify the antitumor effects of ZNF259 in NSCLC, we screened the key signaling factors responsible for modulating their expression, such as FAK, AKT, and MAPK. ${ }^{10,16-22}$ Results revealed that the levels of phosphorylated FAK and AKT were decreased upon ZNF259 overexpression. The decreased level of MMP2 was highly related to the inhibition of PI3K/Akt and ERK, both of which were downstream 
A

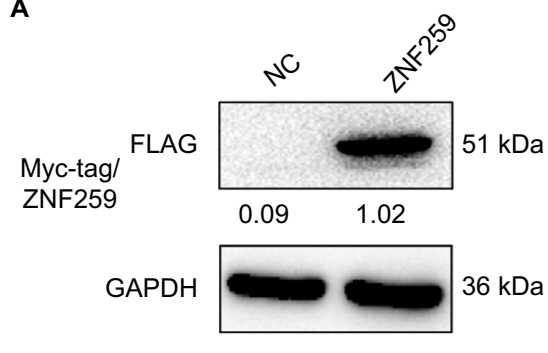

A549

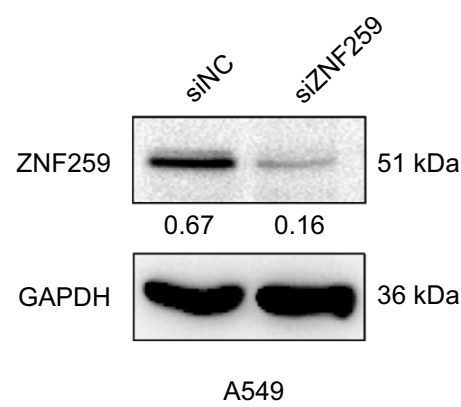

C
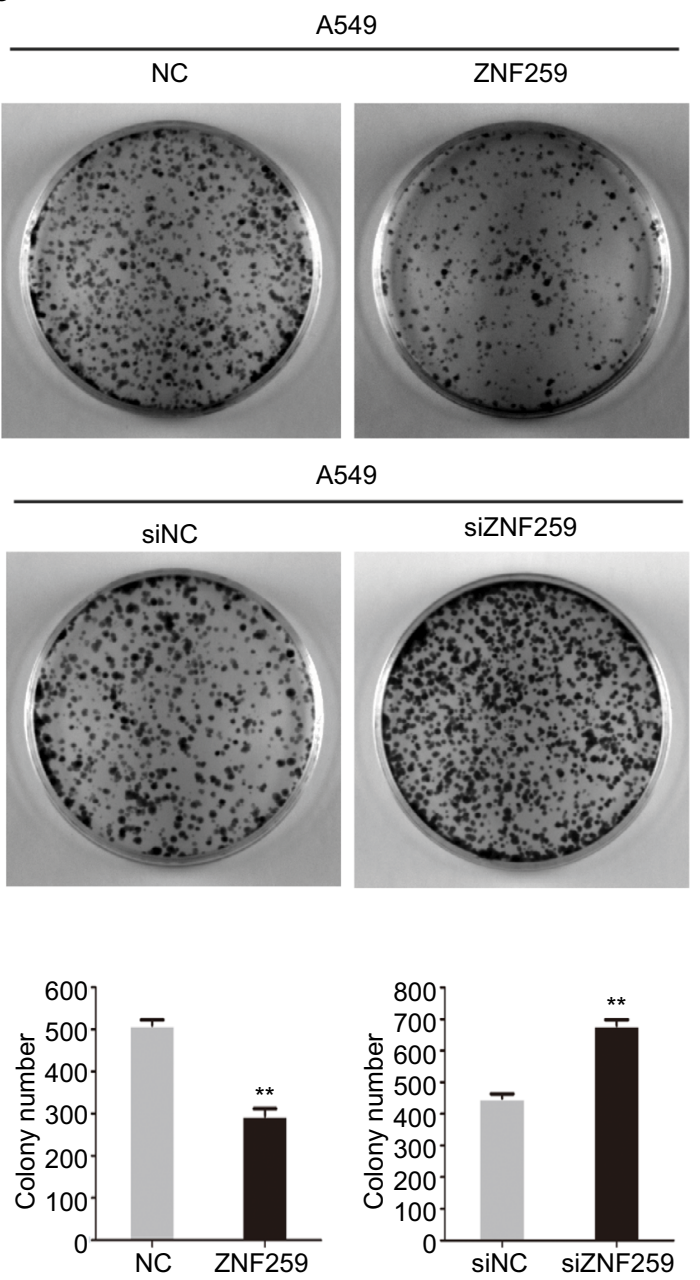
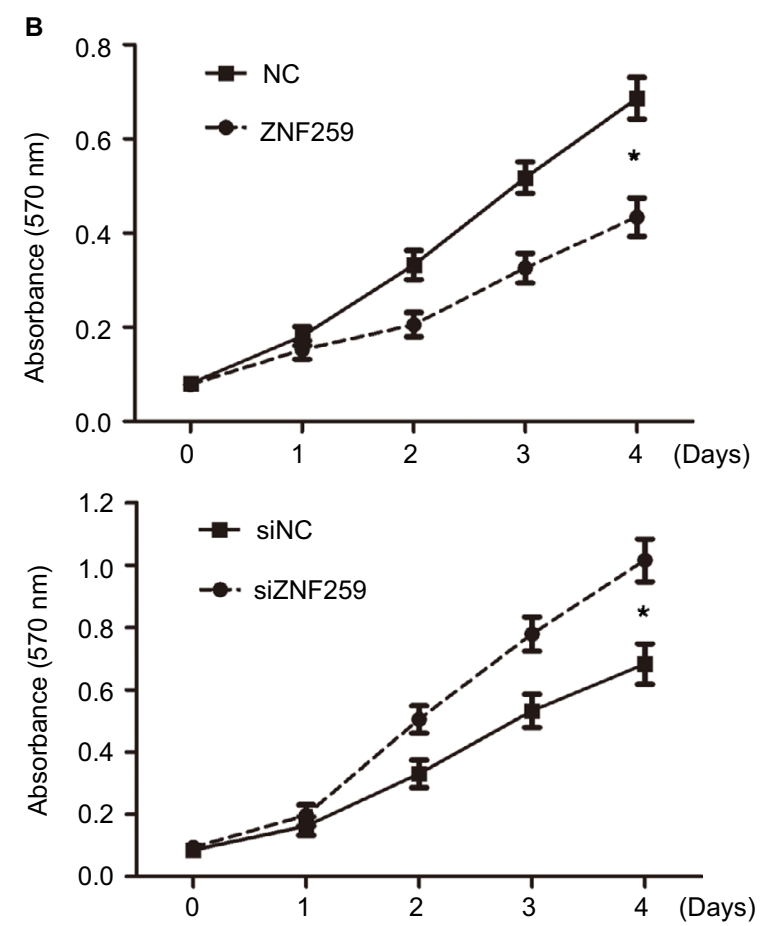

D
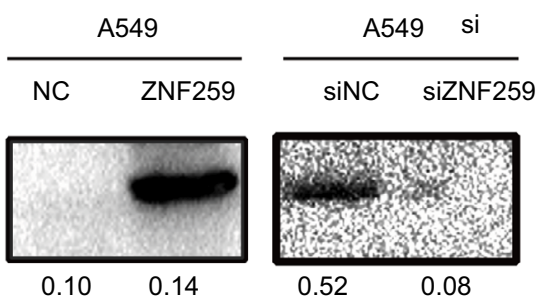

Cyclin D1
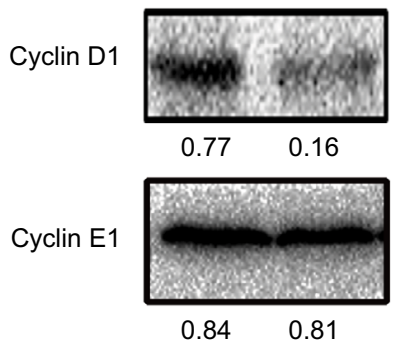

Cyclin E1
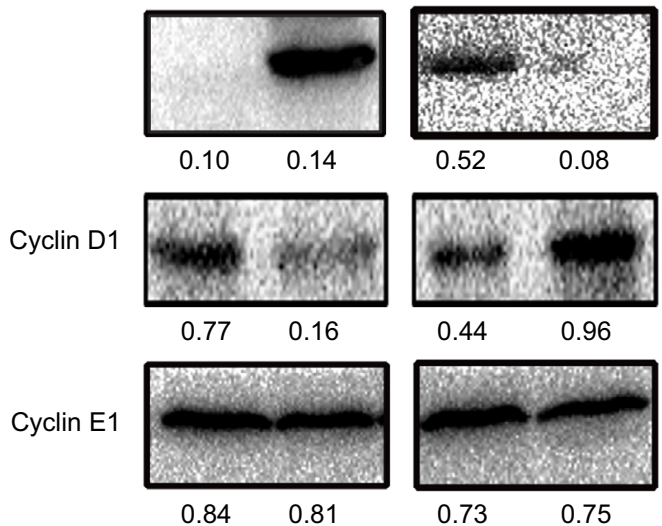

$51 \mathrm{kDa}$
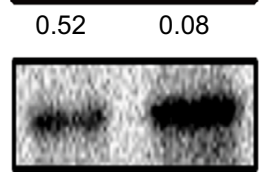

$36 \mathrm{kDa}$
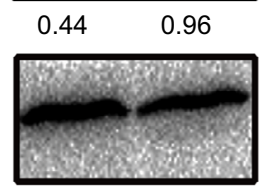

$48 \mathrm{kDa}$

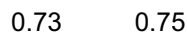

CDK4
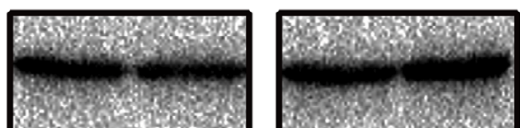

$30 \mathrm{kDa}$
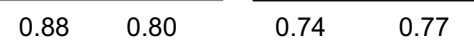

CDK6
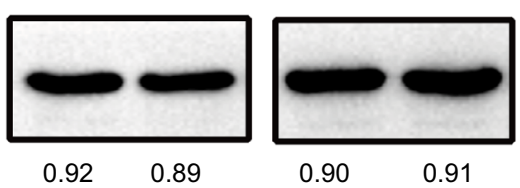

$36 \mathrm{kDa}$

GAPDH

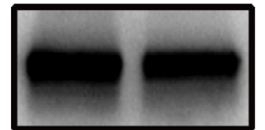

\section{$36 \mathrm{kDa}$}

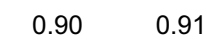

$36 \mathrm{kDa}$

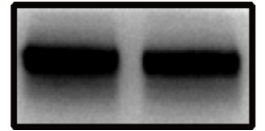

kDa

Figure 2 ZNF259 inhibited proliferation of NSCLC cells.

Notes: (A) Transfection efficiencies after ZNF259 overexpression and depletion by siRNA treatment in A549 cells were determined by Western blotting. (B) The proliferation and (C) colony-formation abilities of A549 cells were reduced after overexpressing ZNF259 and were increased when treated with siRNA targeting ZNF259. (D) Cyclin DI was downregulated and upregulated upon ZNF259 overexpression and depletion in A549 cells, respectively. However, other proteins, such as cyclin EI, CDK4, and CDK6, showed no evident changes in expression. All studies were performed three times. ${ }^{*} P$-value $<0.05$; ${ }^{* * P}$-value $<0.01$.

Abbreviations: NC, negative control; NSCLC, non-small cell lung cancer; si, small interfering; ZNF259, zinc finger protein 259. 

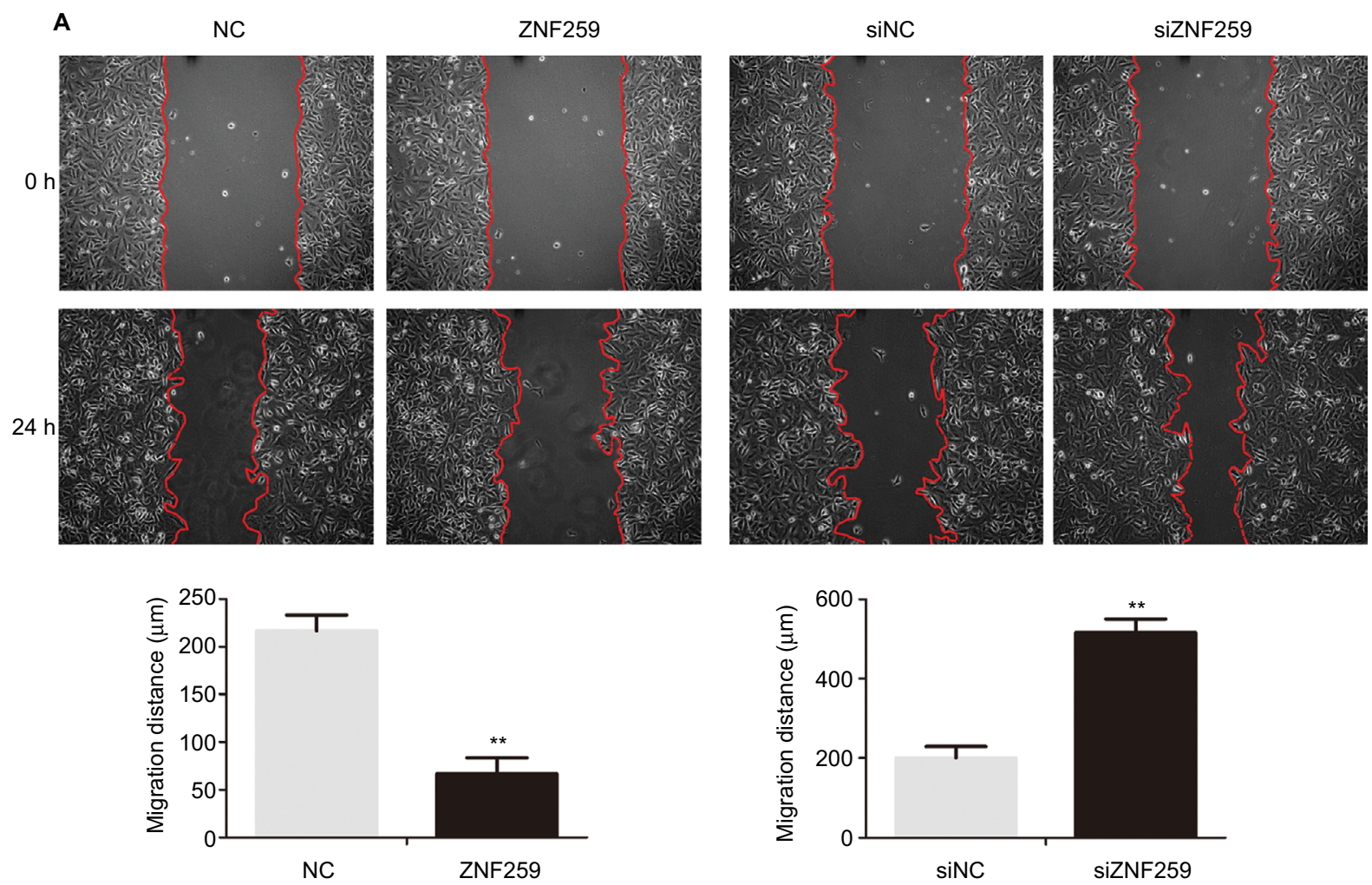

B
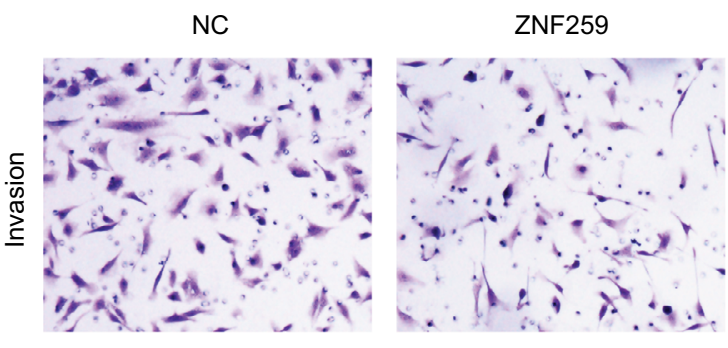

siNC
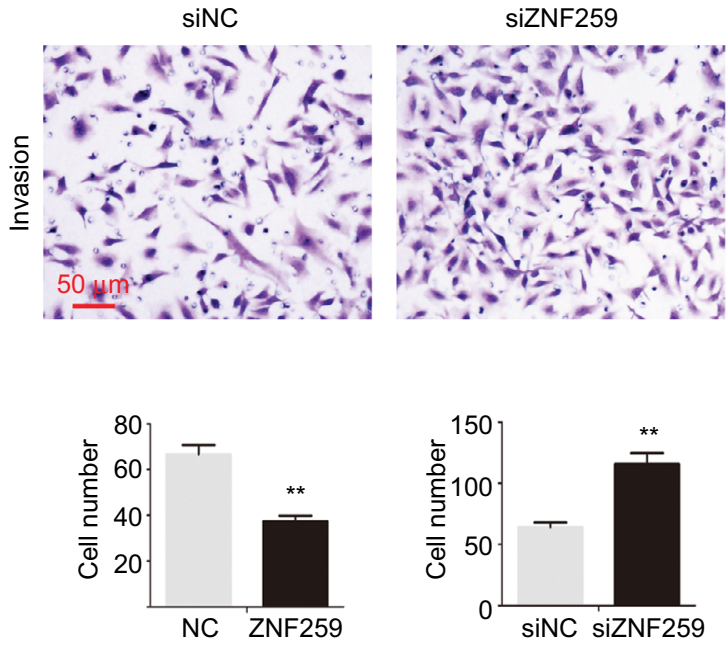

C

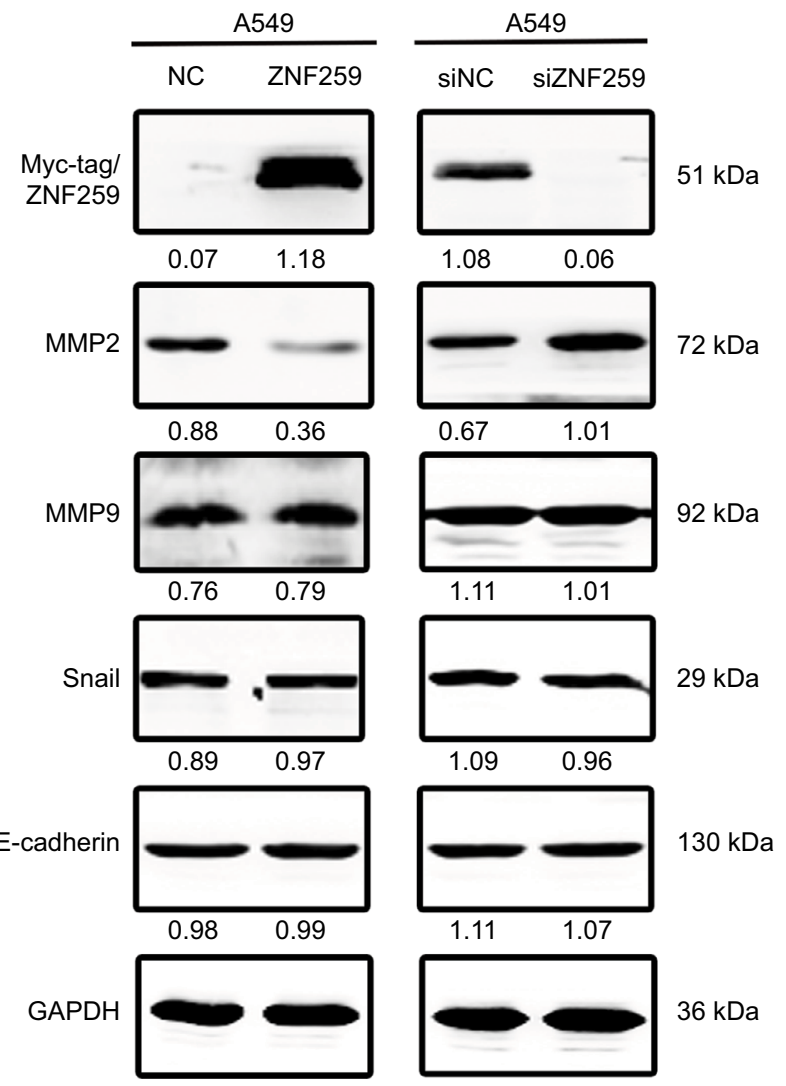

Figure 3 ZNF259 suppressed cell migration and invasion in NSCLC cells.

Notes: ZNF259 overexpression significantly reduced (A) cell migration and (B) invasion in A549 cells. ZNF259 knockdown resulted in enhanced (A) migration and (B) invasion in A549 cells. (C) MMP2 was downregulated or upregulated following ZNF259 overexpression or knockdown in A549 cells, respectively. However, other proteins, such as MMP9, Snail, and E-cadherin, showed no evident changes in expression. All studies were repeated three times.

Abbreviations: NC, negative control; NSCLC, non-small cell lung cancer; si, small interfering; ZNF259, zinc finger protein 259. 
A
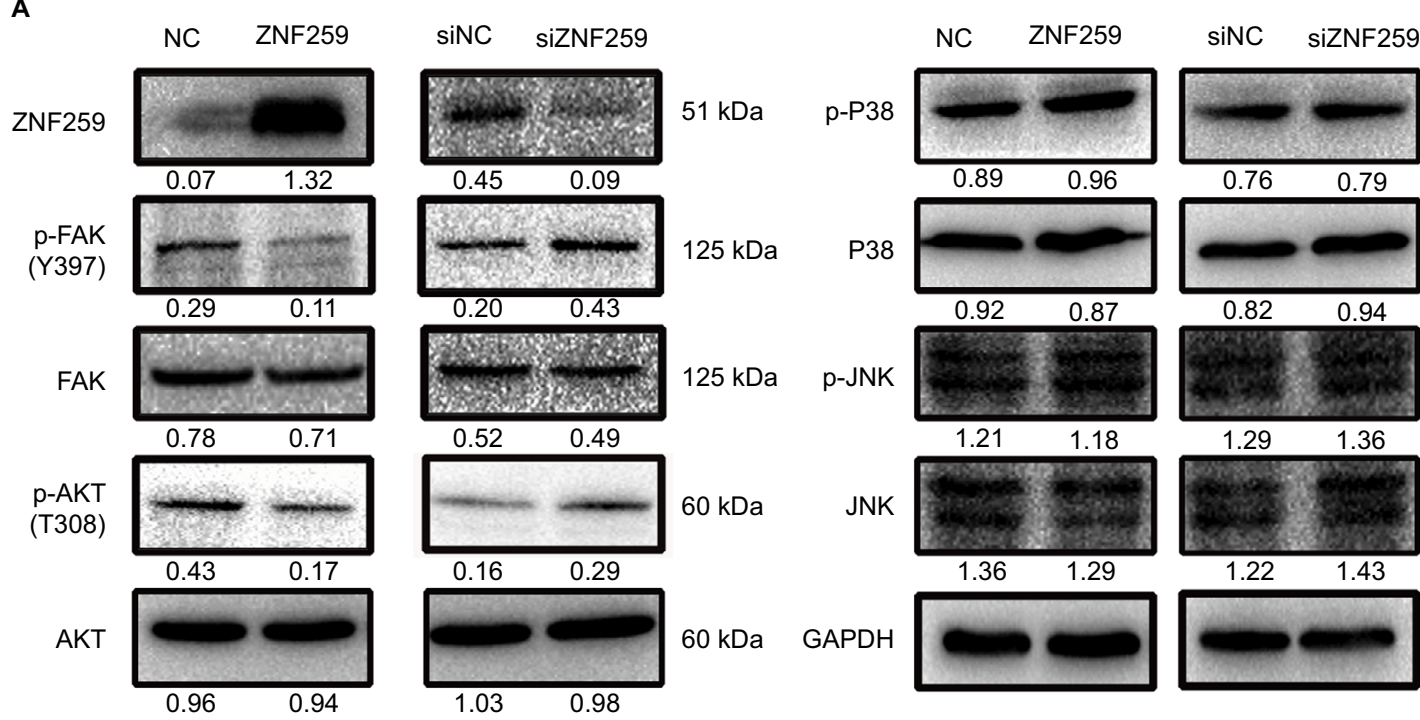

$43 \mathrm{kDa}$

P38
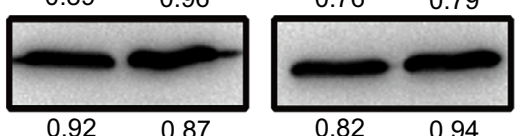

$43 \mathrm{kDa}$
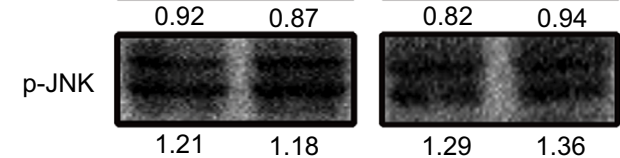

$54 \mathrm{kDa}$

$46 \mathrm{kDa}$

JNK
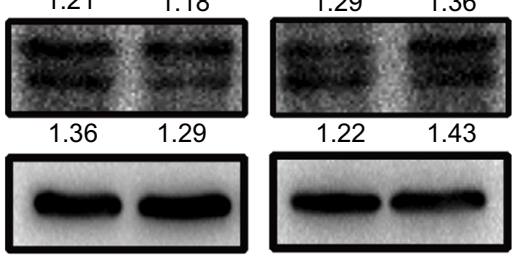

$54 \mathrm{kDa}$

$46 \mathrm{kDa}$

GAPDH

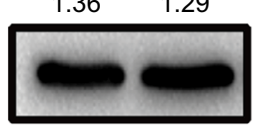

$36 \mathrm{kDa}$

B

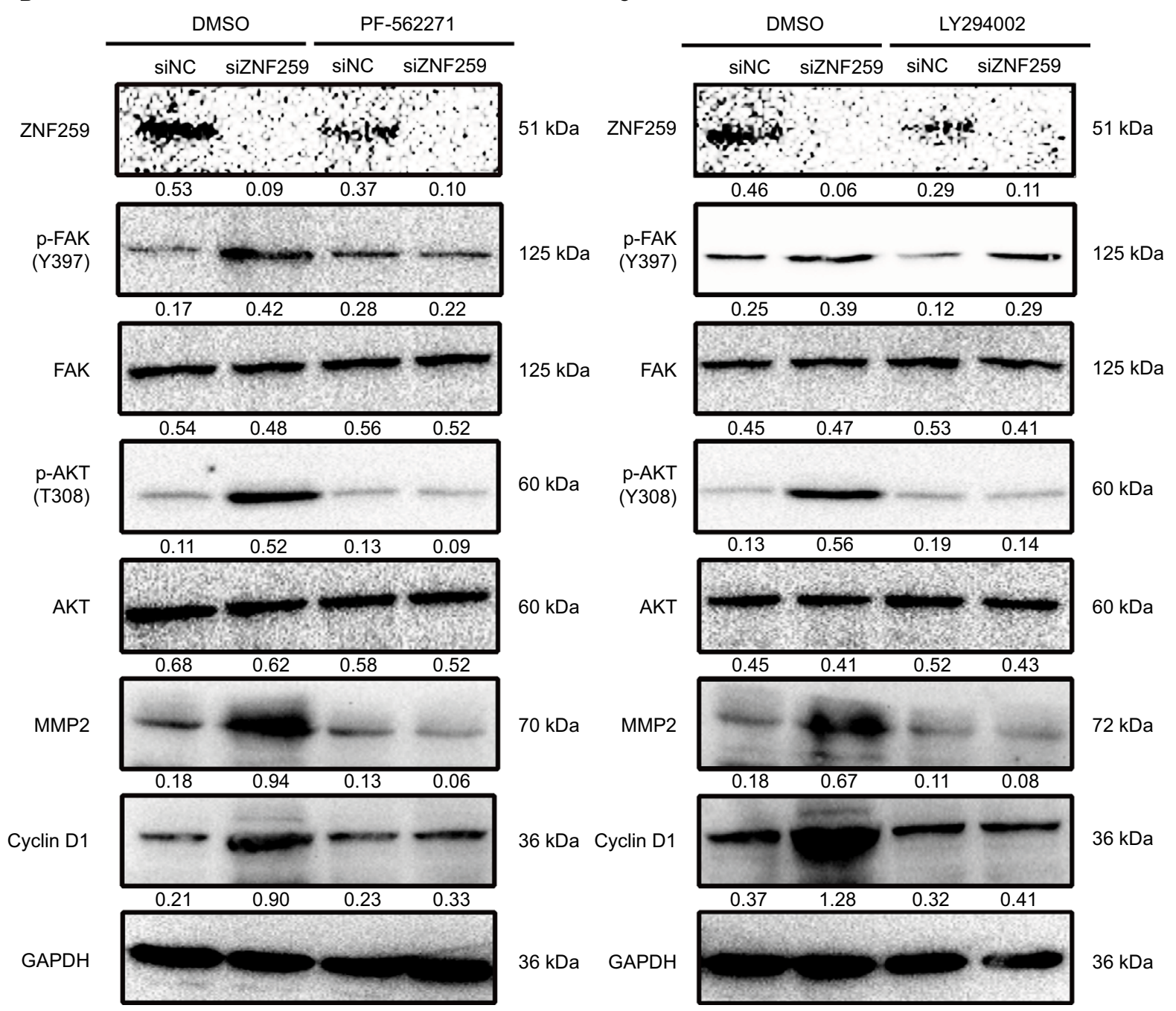

C

Figure 4 ZNF259 reduced cyclin DI and MMP2 levels by inhibiting Fak/Akt phosphorylation.

Notes: (A) The levels of Akt (Thr308), Akt, Fak (Tyr397), Fak, P-JNK (Thr 183/Tyr 185), JNK, p-P38 (Thr 180/Tyr 182), and P38 were determined after ZNF259 overexpression or knockdown in A549 cells. (B) PF56227I, a Fak inhibitor, was added to the culture media of A549 cells with or without ZNF259 knockdown. DMSO was used as negative control. (C) LY294002, an inhibitor of Akt, was added to the culture media of A549 cells with or without ZNF259 depletion. DMSO was used as negative control. All studies were performed three times.

Abbreviations: NC, negative control; NSCLC, non-small cell lung cancer; si, small interfering; ZNF259, zinc finger protein 259. 
targets of FAK in breast cancer cells. ${ }^{23}$ Increase of cyclin D1 could promote cell proliferation by regulating PI3K/AKT signaling. ${ }^{24}$ We next validated the upregulation of MMP2 and cyclin D1 by silencing FAK and AKT signaling. Results showed that MMP2 and cyclin D1 levels were decreased upon addition of PF-562271 and LY294002 to the culture medium after ZNF259 depletion, which indicated that ZNF259 suppressed MMP2 and cyclin D1 expression by FAK-AKT signaling. Previous studies demonstrated the potential role of FAK as an upstream component of the AKT signaling pathway. ${ }^{25-27}$ Our results revealed that in ZNF259-depleted cells, treatment with AKT inhibitor reduced the expression of p-Akt, cyclin D1, and MMP2, but did not affect p-FAK expression. Treatment of ZNF259-depleted cells with FAK inhibitor showed similar effects, but reduced p-Akt levels. Consistent with previous reports, these results also indicated that FAK acts as an upstream factor. ${ }^{25-27}$

In conclusion, ZNF259 was expressed in the cytoplasm of normal lung tissues or cells, but downregulated in lung cancer cells. ZNF259 downregulation was found to be significantly correlated with tumor size, high TNM stage, and positive lymph node metastasis. ZNF259 inhibited MMP2 and cyclin D1 by inactivating FAK-AKT signaling, thereby resulting in reduced invasion and proliferation ability of NSCLC cells.

\section{Acknowledgments}

This study was supported by the Outstanding Scientific Foundation of Shengjing Hospital (number 201210 to Xianghong Yang) and the National Natural Science Foundation of China (number 81402369 to Guiyang Jiang).

\section{Disclosure}

The authors report no conflicts of interest in this work.

\section{References}

1. Siegel RL, Miller KD, Jemal A. Cancer statistics, 2016. CA Cancer J Clin. 2016;66(1):7-30.

2. Soon YY, Stockler MR, Askie LM, Boyer MJ. Duration of chemotherapy for advanced non-small-cell lung cancer: a systematic review and meta-analysis of randomized trials. J Clin Oncol. 2009;27(20): 3277-3283.

3. Gangwani L, Mikrut M, Theroux S, Sharma M, Davis RJ. Spinal muscular atrophy disrupts the interaction of ZPR1 with the SMN protein. Nat Cell Biol. 2001;3(4):376-383.

4. Gangwani L, Flavell RA, Davis RJ. ZPR1 is essential for survival and is required for localization of the survival motor neurons (SMN) protein to Cajal bodies. Mol Cell Biol. 2005;25(7):2744-2756.

5. Galcheva-Gargova Z, Gangwani L, Konstantinov KN, et al. The cytoplasmic zinc finger protein ZPR1 accumulates in the nucleolus of proliferating cells. Mol Biol Cell. 1998;9(10):2963-2971.

6. Gangwani L. Deficiency of the zinc finger protein ZPR1 causes defects in transcription and cell cycle progression. $J$ Biol Chem. 2006;281(52):40330-40340.
7. Travis WD, Brambilla E, Burke AP, Marx A, Nicholson AG. Introduction to the 2015 World Health Organization classification of tumors of the lung, pleura, thymus, and heart. JThorac Oncol. 2015;10(9):1240-1242.

8. Goldstraw P. Updated staging system for lung cancer. Surg Oncol Clin NAm. 2011;20(4):655-666.

9. Bradford MM. A rapid and sensitive method for the quantitation of microgram quantities of protein utilizing the principle of protein-dye binding. Anal Biochem. 1976;72:248-254.

10. Wu W, Liu Q, Liu Y, Yu Z, Wang Y. Dixdc1 targets CyclinD1 and p21 via PI3K pathway activation to promote Schwann cell proliferation after sciatic nerve crush. Biochem Biophys Res Commun. 2016;478(2):956-963.

11. Chai XM, Li YL, Chen H, Guo SL, Shui LL, Chen YJ. Cigarette smoke extract alters the cell cycle via the phospholipid transfer protein/transforming growth factor- $\beta 1 /$ CyclinD1/CDK4 pathway. Eur J Pharmacol. 2016;786:85-93.

12. Park SJ, Kong HK, Kim YS, Lee YS, Park JH. Inhibition of S-adenosylhomocysteine hydrolase decreases cell mobility and cell proliferation through cell cycle arrest. Am J Cancer Res. 2015;5(7):2127-2138.

13. Chen Y, Yu Y, Sun S, et al. Bradykinin promotes migration and invasion of hepatocellular carcinoma cells through TRPM7 and MMP2. Exp Cell Res. 2016;349(1):68-76.

14. Bae GY, Choi SJ, Lee JS, et al. Loss of E-cadherin activates EGFRMEK/ERK signaling, which promotes invasion via the ZEB1/MMP2 axis in non-small cell lung cancer. Oncotarget. 2013;4(12):2512-2522.

15. Galcheva-Gargova Z, Konstantinov KN, Wu IH, Klier FG, Barrett T, Davis RJ. Binding of zinc finger protein ZPR1 to the epidermal growth factor receptor. Science. 1996;272(5269):1797-1802.

16. Luo Y, Liang F, Zhang ZY. PRL1 promotes cell migration and invasion by increasing MMP2 and MMP9 expression through Src and ERK1/2 pathways. Biochemistry. 2009;48(8):1838-1846.

17. Flinder LI, Wierød L, Rosseland CM, Huitfeldt HS, Skarpen E. FAK regulates Cdk2 in EGF-stimulated primary cultures of hepatocytes. $J$ Cell Physiol. 2013;228(6):1304-1313.

18. Tang Y, Lv P, Sun Z, Han L, Zhou W. 14-3-3 $\beta$ Promotes migration and invasion of human hepatocellular carcinoma cells by modulating expression of MMP2 and MMP9 through PI3K/Akt/NF-KB pathway. PLoS One. 2016;11(1):e0146070.

19. Cheng G, Gao F, Sun X, Bi H, Zhu Y. Paris saponin VII suppresses osteosarcoma cell migration and invasion by inhibiting MMP-2/9 production via the p38 MAPK signaling pathway. Mol Med Rep. 2016;14(4): 3199-3205.

20. Recchia AG, Musti AM, Lanzino M, et al. A cross-talk between the androgen receptor and the epidermal growth factor receptor leads to $\mathrm{p} 38 \mathrm{MAPK}$ dependent activation of mTOR and cyclinD1 expression in prostate and lung cancer cells. Int J Biochem Cell Biol. 2009;41(3):603-614.

21. Wang SY, Gao K, Deng DL, et al. TLE4 promotes colorectal cancer progression through activation of JNK/c-Jun signaling pathway. Oncotarget. 2016;7(3):2878-2888.

22. Tian G, Luo X, Tang C, et al. Astrocyte contributes to pain development via MMP2-JNK1/2 signaling in a mouse model of complex regional pain syndrome. Life Sci. 2017;170:64-71.

23. Cao W, Zheng W, Chen T. Ruthenium polypyridyl complex inhibits growth and metastasis of breast cancer cells by suppressing FAK signaling with enhancement of TRAIL-induced apoptosis. Sci Rep. 2015;5:9157.

24. Zhang W, Lei C, Fan J, Wang J. miR-18a promotes cell proliferation of esophageal squamous cell carcinoma cells by increasing cylin D1 via regulating PTEN-PI3K-AKT-mTOR signaling axis. Biochem Biophys Res Commun. 2016;477(1):144-149.

25. Jia D, Zhu Q, Liu H, et al. Osteoprotegerin disruption attenuates HySuinduced pulmonary hypertension through integrin $\alpha v \beta 3 / F A K / A K T$ pathway suppression. Circ Cardiovasc Genet. 2017;10(1): e001591.

26. Wang Z, Wang Z, Li G, et al. CXCL1 from tumor-associated lymphatic endothelial cells drives gastric cancer cell into lymphatic system via activating integrin $\beta 1 /$ FAK/AKT signaling. Cancer Lett. 2017;385:28-38.

27. Leng $C$, Zhang ZG, Chen WX, et al. An integrin beta4-EGFR unit promotes hepatocellular carcinoma lung metastases by enhancing anchorage independence through activation of FAK-AKT pathway. Cancer Lett. 2016;376(1):188-196. 
Cancer Management and Research

\section{Publish your work in this journal}

Cancer Management and Research is an international, peer-reviewed open access journal focusing on cancer research and the optimal use of preventative and integrated treatment interventions to achieve improved outcomes, enhanced survival and quality of life for the cancer patient.

The manuscript management system is completely online and includes

Submit your manuscript here: https://www.dovepress.com/cancer-management-and-research-journal 